\title{
FINITE DIMENSIONALITY OF IRREDUCIBLE UNITARY REPRESENTATIONS OF COMPACT QUANTUM GROUPS
}

\author{
XIU-CHI QUAN
}

(Communicated by Palle E. T. Jorgensen)

\begin{abstract}
In this paper, we study the representations of Hopf $C^{*}$-algebras; the main result is that every irreducible left unitary representation of a Hopf $C^{*}$-algebra with a Haar measure is finite dimensional. To prove this result, we first study the comodule structure of the space of Hilbert-Schmidt operators; then we use this comodule structure to show that every irreducible left unitary representation of a Hopf $C^{*}$-algebra with a Haar measure is finite dimensional.
\end{abstract}

\section{INTRODUCTION}

It is well known that, for compact groups, every irreducible unitary representation is finite dimensional. For a simple proof about this result, we refer to [N]. In this paper, we will generalize this result to Hopf $C^{*}$-algebras with Haar measures; namely, we will prove that every irreducible left unitary representation of a Hopf $C^{*}$-algebra with a Haar measure is finite dimensional. To prove this result, we first study the comodule structure of Hilbert-Schmidt operators; then we use the result about Hilbert-Schmidt operators to show that every irreducible left unitary representation is finite dimensional.

In earlier work in this direction, Woronowicz [W] proved that every irreducible unitary representation of a compact matrix quantum group is finite dimensional; the author [Q] showed that for a Hopf $C^{*}$-algebra with the PeterWeyl property, every irreducible unitary representation is finite dimensional. The previous approach depends heavily on the Peter-Weyl property. Here we are going to generalize these results; the method we use is elementary, which does not use the Peter-Weyl property.

Before we turn to the contents of the paper, let us recall the definitions of Hopf $C^{*}$-algebras, representations, and Haar measures of Hopf $C^{*}$-algebras.

Let $A$ be a $C^{*}$-algebra with a dense *-subalgebra $\mathscr{A}$ and $\Phi: A \rightarrow A \otimes A$ a

Received by the editors September 28, 1992 and, in revised form, October 19, 1992. 1991 Mathematics Subject Classification. Primary 46L89, 47A67; Secondary 22D25.

Key words and phrases. Hilbert-Schmidt operator, Hopf $C^{*}$-algebra, Haar measure. 
$C^{*}$-homomorphism. We say that $(A, \Phi)$ is a Hopf $C^{*}$-algebra if the diagram

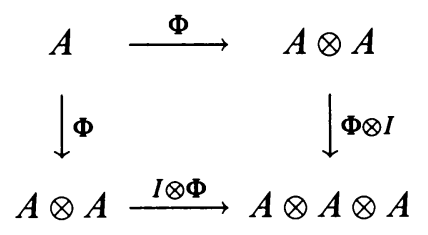

commutes and $\Phi(\mathscr{A}) \subset \mathscr{A} \otimes \mathscr{A}$, where $\Phi$ is the comultiplication of $(A, \Phi)$. If $A$ is a von Neumann algebra, we call $A$ a Hopf-von Neumann algebra. By an involution of $(A, \Phi)$, we mean a ${ }^{*}$-anti-isomorphism $k: \mathscr{A} \rightarrow \mathscr{A}$ such that the diagram

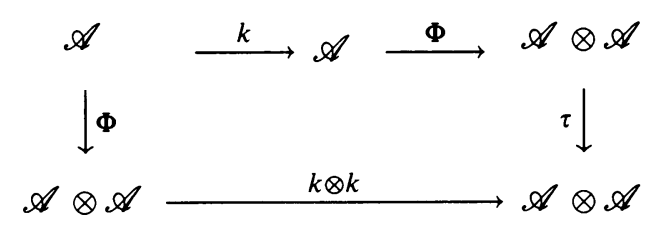

commutes where $\tau: A \otimes A \rightarrow A \otimes A$ is the flip automorphism and $\tau(a \otimes b)=$ $b \otimes a, \forall a, b \in A$.

We say that $e: \mathscr{A} \rightarrow C$ is a counit of $A$ if $e$ is a $C^{*}$-homomorphism and the following diagram commutes:

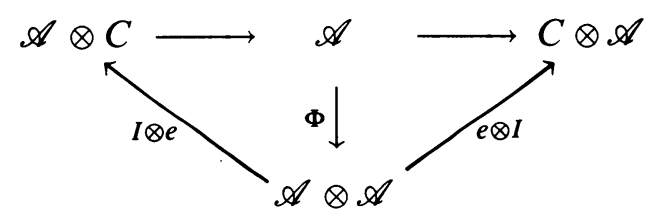

We say that $(A, \Phi, k, e)$ is a compact quantum group if the following diagrams commute:

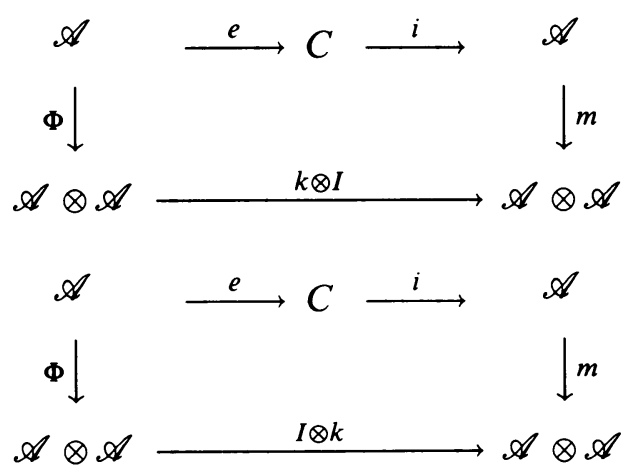

Let $A$ be a coalgebra and $M$ a linear space. Let $\psi: M \rightarrow A \otimes M$ be a linear 
map which makes the following diagrams commute:

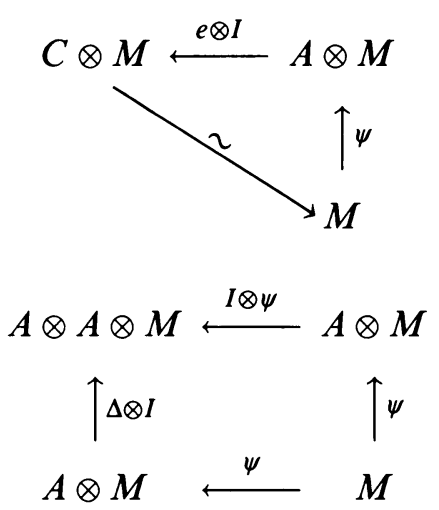

The pair $(M, \psi)$ is called a unital left $A$-comodule; if only the second diagram commutes, we call $(M, \psi)$ a left $A$-comodule, and $\psi$ is said to be its structure map. A right $A$-comodule can be defined similarly. For more information about comodules, we refer to [A].

Let $M$ be a left $A$-comodule with structure map $\psi$; a subspace $M_{1} \subset M$ is said to be left invariant if $\psi\left(M_{1}\right) \subset A \otimes M_{1}$. We say that $M$ is an irreducible left $A$-comodule if $M$ is the only nonzero left invariant subspace of $M$.

Now let $(A, \Phi)$ be a Hopf $C^{*}$-algebra with unit and $\mathscr{A}$ the dense *subalgebra of $A$. We say that $V$ is a left $A$-comodule if it is a left $\mathscr{A}$ comodule which is defined as above. Suppose that $V$ is a finite-dimensional left $A$-comodule with structure map $\psi: V \rightarrow \mathscr{A} \otimes V$. If $\left\{e_{i}\right\}_{i=1}^{n}$ is a basis of $V$ and

$$
\psi\left(e_{i}\right)=\sum_{k=1}^{n} a_{i k} \otimes e_{k},
$$

the matrix $\left(a_{i j}\right)$ is called the coefficient matrix of $\psi$ with respect to $\left\{e_{i}\right\}_{i=1}^{n}$. Then the comodule property implies that

$$
\Phi\left(a_{i j}\right)=\sum_{k} a_{i k} \otimes a_{k j}
$$

Now suppose that $V$ is a left $A$-comodule, which is also a Hilbert space, with structure map $L: V \rightarrow \mathscr{A} \otimes V$ and inner product $(\cdot, \cdot): V \times V \rightarrow C$. We can extend $(\cdot, \cdot)$ to $\langle\cdot, \cdot\rangle:(\mathscr{A} \otimes V) \times(\mathscr{A} \otimes V) \rightarrow \mathscr{A}$ as

$$
\langle a \otimes x, b \otimes y\rangle=a b^{*}(x, y), \quad \forall a, b \in \mathscr{A}, x, y \in V .
$$

A left unitary representation $\pi$ of $A$ on a Hilbert space $H$ is a comodule map from $H$ into $\mathscr{A} \otimes H$ such that

$$
\langle\pi(x), \pi(y)\rangle=\langle x, y\rangle, \quad \forall x, y \in H .
$$

Let $(A, \Phi, k, e)$ be a Hopf $C^{*}$-algebra and $\sigma$ a positive linear functional. We say that $\sigma$ is a left Haar measure if, for all $x^{*} \in A^{*}$, we have

$$
x^{*} \cdot \sigma=\left\langle x^{*}, I\right\rangle \sigma .
$$

Similarly, we can define a right Haar measure.

Note that for a left (right) Haar measure $\sigma$, we have $\sigma(k(a))=\sigma(a), \forall a \in$ $\mathscr{A}$. For a proof of this result, we refer to [W]. 
Finally, we come to the contents of this paper. In $\S 2$ we study the comodule structure of Hilbert-Schmidt operators. The main results in this section are that, for any two Hilbert spaces $H_{1}, H_{2}$, which are left unitary left $\mathscr{A}$ comodules, where $\mathscr{A}$ is the dense ${ }^{*}$-subalgebra of a Hopf $C^{*}$-algebra, the space $\operatorname{Hom}_{2}\left(H_{1}, H_{2}\right)$ of Hilbert-Schmidt operators from $H_{1}$ to $H_{2}$ has a natural right $\mathscr{A}$-comodule structure. Also we give a characterization that under what condition a Hilbert-Schmidt operator is a comodule map. In $\S 3$ we first show that the space of Hilbert-Schmidt operators is invariant under the action of a Haar measure. Then we use the results in $\S 2$ to show that every irreducible left unitary representation of a Hopf $C^{*}$-algebra with a Haar measure is finite dimensional.

\section{COMOdUle STRUCTURE FOR HilberT-SCHMidT OPERATORS}

In this section, we are going to endow a comodule structure to the space of Hilbert-Schmidt operators between two Hilbert spaces which are left unitary comodules of a Hopf $C^{*}$-algebra.

Let $H_{1}, H_{2}$ be Hilbert spaces. An operator $T \in B\left(H_{1}, H_{2}\right)$ is said to be a Hilbert-Schmidt operator if, for one orthonormal basis $\left(e_{i}^{1}\right) \subset H_{1}, \sum_{i}\left\|T e_{i}^{1}\right\|^{2}<$ $\infty$. Let $\operatorname{Hom}_{2}\left(H_{1}, H_{2}\right)$ denote the space of all Hilbert-Schmidt operators from $H_{1}$ to $H_{2}$. For every $T \in H_{2}\left(H_{2}, H_{2}\right)$, let

$$
\|T\|_{2}^{2}=\sum_{i}\left\|T e_{i}^{1}\right\|^{2} .
$$

It is well known that $\left(\operatorname{Hom}_{2}\left(H_{1}, H_{2}\right),\|\cdot\|_{2}\right)$ is a Hilbert space with the inner product given by

$$
\left\langle T_{1}, T_{2}\right\rangle=\sum_{i}\left\langle T_{1} e_{i}^{1}, T_{2} e_{i}^{1}\right\rangle .
$$

Now let $(A, \Phi, k, e)$ be a Hopf $C^{*}$-algebra with a dense *-subalgebra $\mathscr{A}$. Suppose that $H_{1}, H_{2}$ are also left unitary $\mathscr{A}$-comodules with structure maps $\psi_{1}$ and $\psi_{2}$. Fix orthonormal bases $\left\{e_{i}^{1}\right\},\left\{e_{j}^{2}\right\}$ for $H_{1}, H_{2}$ respectively. Suppose that

$$
\psi_{1}\left(e_{i}^{1}\right)=\sum_{k} a_{i k}^{1} \otimes e_{k}^{1}, \quad \psi_{2}\left(e_{j}^{2}\right)=\sum_{s} a_{j s}^{2} \otimes e_{s}^{2} .
$$

Let $H_{1}^{\prime}$ be the complex conjugate of $H_{1}$. Then $\left\{e_{i}^{1}\right\}$ also form an orthonormal basis for $H_{1}^{\prime}$. It is straightforward to verify that

$$
\psi_{1}^{\prime}\left(e_{i}^{1}\right)=\sum_{k} e_{k}^{1} \otimes a_{k i}^{1}
$$

gives $H_{1}^{\prime}$ a right $\mathscr{A}$-comodule structure.

Since $\operatorname{Hom}_{2}\left(H_{1}, H_{2}\right) \cong H_{1}^{\prime} \hat{\otimes} H_{2}$ (projective tensor product), define

$$
\psi_{H_{1}, H_{2}}: \operatorname{Hom}_{2}\left(H_{1}, H_{2}\right) \rightarrow \operatorname{Hom}_{2}\left(H_{1}, H_{2}\right) \otimes \mathscr{A}
$$

as

$$
\psi_{H_{1}, H_{2}}\left(e_{i}^{1} \otimes e_{j}^{2}\right)=\sum_{k, s}\left(e_{k}^{1} \otimes e_{s}^{2}\right) \otimes a_{k i}^{1} k\left(a_{j s}^{2}\right)
$$


Proposition 2.1. With the notation as above, $\left(\operatorname{Hom}_{2}\left(H_{1}, H_{2}\right), \psi_{H_{1}, H_{2}}\right)$ is a right $\mathscr{A}$-comodule with structure map $\psi_{\mathrm{H}_{1}, \mathrm{H}_{2}}$.

Proof. We only need to verify that the following diagram commutes:

$$
\begin{aligned}
& \operatorname{Hom}_{2}\left(H_{1}, H_{2}\right) \quad \stackrel{\psi_{H_{1}, H_{2}}}{\longrightarrow} \operatorname{Hom}_{2}\left(H_{1}, H_{2}\right) \otimes \mathscr{A} \\
& \downarrow \psi_{H_{1}, H_{2}} \quad \downarrow \psi_{H_{1}, H_{2} \otimes I} \\
& \operatorname{Hom}_{2}\left(H_{1}, H_{2}\right) \otimes \mathscr{A} \stackrel{I \otimes \Phi}{\longrightarrow} \operatorname{Hom}_{2}\left(H_{1}, H_{2}\right) \otimes \mathscr{A} \otimes \mathscr{A}
\end{aligned}
$$

In fact, for $e_{i}^{1} \otimes e_{j}^{2}$, we have

$$
\begin{aligned}
\left(\psi_{H_{1}, H_{2}} \otimes I\right) \psi_{H_{1}, H_{2}}\left(e_{i}^{1} \otimes e_{j}^{2}\right) & =\sum_{k, s} \psi_{H_{1}, H_{2}}\left(e_{k}^{1} \otimes e_{s}^{2}\right) \otimes a_{k i}^{1} k\left(a_{j s}^{2}\right) \\
& =\sum_{k, s} \sum_{p, q}\left(e_{p}^{1} \otimes e_{q}^{2}\right) \otimes a_{p k}^{1} k\left(a_{s q}^{2}\right) \otimes a_{k i}^{1} k\left(a_{j s}^{2}\right) .
\end{aligned}
$$

On the other hand, we have

$$
\begin{aligned}
(I \otimes \Phi) \psi_{H_{1}, H_{2}}\left(e_{i}^{1} \otimes e_{j}^{2}\right) & =\sum_{k, s}\left(e_{k}^{1} \otimes e_{s}^{2}\right) \Phi\left(a_{k i}^{1} k\left(a_{j s}^{2}\right)\right) \\
& =\sum_{k, s} \sum_{p, q}\left(e_{p}^{1} \otimes e_{q}^{2}\right) \otimes a_{p k}^{1} k\left(a_{s q}^{2}\right) \otimes a_{k i}^{1} k\left(a_{j s}^{2}\right) .
\end{aligned}
$$

This completes the proof.

For $T \in \operatorname{Hom}_{2}\left(H_{1}, H_{2}\right), v \in H_{1}$, we have

$$
\left(\psi_{H_{1}, H_{2}} T\right)(v)=\tau(m \otimes I)(I \otimes k \otimes I)\left(I \otimes \psi_{2}\right)(I \otimes T)\left(\psi_{1}(v)\right) .
$$

The main result of this section is

Theorem 2.2. With the above assumption and for $T \in \operatorname{Hom}_{2}\left(H_{1}, H_{2}\right)$, the following are equivalent:

(1) $T$ is a left $\mathscr{A}$-comodule map,

(2) $T$ is a right $A^{*}$-module map,

(3) $\psi_{H_{1}, H_{2}}(T)=T \otimes I$,

(4) $x^{*} \cdot T=x^{*}(I) T, \forall x^{*} \in A^{*}$.

Proof. The equivalence between (1) and (2) and (3) and (4) is well known. So we only need to prove the equivalence of (1) and (3).

$(1) \Rightarrow(3)$. Suppose that $T$ is a left $\mathscr{A}$-comodule map. Then

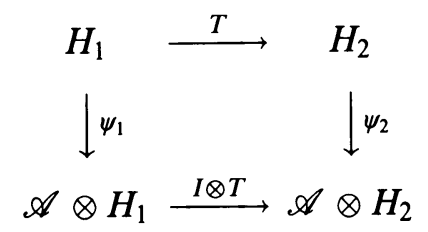

commutes. So, for any $e_{i}^{1} \in H_{1}$, we have

$$
\psi_{2}\left(T e_{i}^{1}\right)=(I \otimes T) \psi_{1}\left(e_{i}^{1}\right),
$$


i.e.,

$$
\begin{aligned}
\psi_{2}\left(T e_{i}^{1}\right) & =\sum_{k}(I \otimes T)\left(a_{i k}^{1} \otimes e_{k}^{1}\right)=\sum_{k} a_{i k}^{1} \otimes T e_{k}^{1}, \\
\left(\psi_{H_{1}, H_{2}} T\right)\left(e_{i}^{1}\right) & =\tau(m \otimes I)(I \otimes k \otimes I)\left(I \otimes \psi_{2}\right)(I \otimes T) \psi_{1}\left(e_{i}^{1}\right) \\
& =\tau(m \otimes I)(I \otimes k \otimes I)\left(I \otimes \psi_{2}\right)\left(\sum_{k} a_{i k}^{1} \otimes T e_{k}^{1}\right) \\
& =\tau(m \otimes I)(I \otimes k \otimes I)\left(\sum_{k, s} a_{i k}^{1} \otimes a_{k s}^{1} \otimes T e_{s}^{1}\right) \\
& =\sum_{k, s} T e_{s}^{1} \otimes a_{i k}^{1} k\left(a_{k s}^{1}\right)=\sum_{s} T e_{s}^{1} \otimes e\left(a_{i s}^{1}\right) \\
& =T\left(\sum_{s} e\left(a_{i s}^{1}\right) e_{s}^{1}\right) \otimes I=T\left(e_{i}^{1}\right) \otimes I .
\end{aligned}
$$

So $\psi_{H_{1}, H_{2}}(T)=T \otimes I$. have

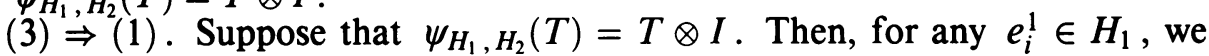

$$
\left(\psi_{H_{1}, H_{2}} T\right)\left(e_{i}^{1}\right)=T\left(e_{i}^{1}\right) \otimes I .
$$

Let $T e_{i}^{1}=\sum_{p} b_{i p} e_{p}^{2}$. So we have

$$
\begin{aligned}
\left(\psi_{H_{1}, H_{2}} T\right)\left(e_{i}^{1}\right) & =\tau(m \otimes I)(I \otimes k \otimes I)\left(I \otimes \psi_{2}\right)\left(\sum_{k} a_{i k}^{1} \otimes T e_{k}^{1}\right) \\
& =\tau(m \otimes I)(I \otimes k \otimes I)\left(\sum_{k} a_{i k}^{1} \otimes \psi_{2}\left(T e_{k}^{1}\right)\right) \\
& =\sum_{k, p} b_{k p} \tau(m \otimes I)(I \otimes k \otimes I)\left(a_{i k}^{1} \otimes \sum_{s} a_{p s}^{2} \otimes e_{s}^{2}\right) \\
& =\sum_{k, p, s} b_{k p} e_{s}^{2} \otimes a_{i k}^{1} k\left(a_{p s}^{2}\right) .
\end{aligned}
$$

Thus

$(m \otimes I)(2,3,1)\left(\psi_{2} \otimes I\right)\left(\psi_{H_{1}, H_{2}} T\right)\left(e_{i}^{1}\right)$

$$
\begin{aligned}
& =(m \otimes I)(2,3,1)\left(\sum_{k, p, s, t} b_{k p} a_{s t}^{2} \otimes e_{t}^{2} \otimes a_{i k}^{1} k\left(a_{p s}^{2}\right)\right) \\
& \left.=(m \otimes I)\left(\sum_{k, p, s, t} b_{k p} a_{i k}^{1} k\left(a_{p s}^{2}\right) \otimes a_{s t}^{2} \otimes e_{t}^{2}\right)=\sum_{k, p, s, t} b_{k p} a_{i k}^{1} k\left(a_{p s}^{2}\right) a_{s t}^{2} \otimes e_{t}^{2}\right) \\
& =\sum_{k, p, t} b_{k p} e\left(a_{p t}^{2}\right) a_{i k}^{1} \otimes e_{t}^{2}=\sum_{k, p} a_{i k}^{1} \otimes b_{k p} e_{p}^{2}=\sum_{k} a_{i k}^{1} \otimes T e_{k}^{1},
\end{aligned}
$$

where $(2,3,1)$ is the map from $\mathscr{A} \otimes H \otimes \mathscr{A}$ into $\mathscr{A} \otimes \mathscr{A} \otimes H$ such that 
$(2,3,1)(a \otimes h \otimes b)=b \otimes a \otimes h, \forall a, b \in \mathscr{A}, h \in H$. On the other hand,

$(m \otimes I)(2,3,1)\left(\psi_{2} \otimes I\right)\left(T e_{i}^{1} \otimes I\right)=(m \otimes I)(2,3,1)\left(\sum_{p} b_{i p} \psi_{2}\left(e_{p}^{2}\right) \otimes I\right)$

$$
=\sum_{p, s} b_{i p} a_{p s}^{2} \otimes e_{s}^{2}=\psi_{2}\left(T e_{i}^{1}\right)
$$

Thus we have that $\psi_{2}\left(T e_{i}^{1}\right)=\sum_{k} a_{i k}^{1} \otimes T e_{k}^{1}$, i.e., $T$ is a comodule map. This completes the proof.

\section{FINITE DIMENSIONALITY OF IRREDUCIBLE UNITARY REPRESENTATIONS}

In this section, we are going to show that every irreducible unitary representation of a Hopf $C^{*}$-algebra with a Haar measure is finite dimensional. We begin the section with the following result, which is the consequence of Theorem 2.2.

Proposition 3.1. With the same notation as above, if $T \in \operatorname{Hom}_{2}\left(H_{1}, H_{2}\right)$, then $\sigma \cdot T \in \operatorname{Hom}_{2}\left(H_{1}, H_{2}\right)$.

Proof. Since $T=\sum_{i, j} b_{i j} e_{i}^{1} \otimes e_{j}^{2}$, where $\sum_{i, j}\left|b_{i j}\right|^{2}<\infty$,

$$
\begin{aligned}
\sigma \cdot T & =(I \otimes \sigma) \psi_{H_{1}, H_{2}}(T) \\
& =(I \otimes \sigma)\left[\sum_{i, j} b_{i j} \psi_{H_{1}, H_{2}}\left(e_{i}^{1} \otimes e_{j}^{2}\right)\right] \\
& =(I \otimes \sigma)\left[\sum_{i, j} b_{i j} \sum_{k, s}\left(e_{k}^{1} \otimes e_{s}^{2}\right) a_{k i}^{1} k\left(a_{j s}^{2}\right)\right] \\
& =\sum_{k, s}\left[\sum_{i, j} b_{i j} \sigma\left(a_{k i}^{1} k\left(a_{j s}^{2}\right)\right)\right] e_{k}^{1} \otimes e_{s}^{2} .
\end{aligned}
$$

But

$$
\begin{gathered}
\sum_{k, s} \sum_{i, j}\left|b_{i j}\right|^{2}\left|\sigma\left(a_{k i}^{1} k\left(a_{j s}\right)\right)\right|^{2} \leq \sum_{k, s} \sum_{i, j}\left|b_{i j}\right|^{2} \sigma\left(a_{k i}^{1}\left(a_{k i}^{1}\right)^{*}\right) \sigma\left(a_{j s}^{2}\left(a_{j s}^{2}\right)^{*}\right) \\
=\sum_{i, j}\left|b_{i j}\right|^{2} \sigma\left(\sum_{k} a_{k i}^{1}\left(a_{k i}^{1}\right)^{*}\right) \sigma\left(\sum_{s} a_{j s}^{2}\left(a_{j s}^{2}\right)^{*}\right)=\sum_{i, j}\left|b_{i, j}\right|^{2},
\end{gathered}
$$

since $H_{1}, H_{2}$ are unitary $\mathscr{A}$-comodules. Hence $\sigma \cdot T \in \operatorname{Hom}_{2}\left(H_{1}, H_{2}\right)$. This finishes the proof.

Lemma 3.2. If $\sigma$ is a left Haar measure on $\mathscr{A}$, then, for any $T \in \operatorname{Hom}_{2}\left(H_{1}, H_{2}\right)$, $\sigma \cdot T$ is a left $\mathscr{A}$-comodule map.

Proof. It is a direct consequence of Theorem 2.2 and the definition of a Haar measure.

Theorem 3.3. If $(A, \Phi, k, e)$ is a Hopf $C^{*}$-algebra with a dense subalgebra $\mathscr{A}$ and a Haar measure $\sigma$, then every irreducible left unitary representation is finite dimensional. 
Proof. Suppose that $H$ is an irreducible left unitary $\mathscr{A}$-comodule with structure map $\pi$. Choose an orthonormal basis $\left\{e_{i}\right\}_{i \in I}$ for $H$. Let $\pi\left(e_{i}\right)=$ $\sum_{j} a_{i j} \otimes e_{j}$. Then, for any $i, j, p, q \in I$, we have

$$
\begin{aligned}
\left\langle e_{i}, \sigma \cdot\left(e_{p} \otimes e_{q}\right) e_{j}\right\rangle & =\left\langle e_{i},(I \otimes \sigma) \psi_{H, H}\left(e_{p} \otimes e_{q}\right) e_{j}\right\rangle \\
& =\left\langle e_{i},(I \otimes \sigma)\left[\sum_{k, s}\left(e_{k} \otimes e_{s}\right) a_{p k} k\left(a_{q s}\right)\right] e_{j}\right\rangle \\
& =\sum_{k, s} \sigma\left(a_{p k} k\left(a_{q s}\right)\right)\left\langle e_{j}, e_{k}\right\rangle\left\langle e_{s}, e_{i}\right\rangle \\
& =\sigma\left(a_{p j} k\left(a_{q i}\right)\right) .
\end{aligned}
$$

Since $\pi$ is a left unitary representation, there exist $i, j, p, q \in I$ such that $\sigma\left(a_{p j} k\left(a_{q i}\right)\right) \neq 0$. Also, $e_{p} \otimes e_{q} \in \operatorname{Hom}_{2}(H, H)$, so $\sigma \cdot\left(e_{p} \otimes e_{q}\right) \in \operatorname{Hom}_{2}(H, H)$. Because $\sigma \cdot\left(e_{p} \otimes e_{q}\right)$ is a left comodule map and $H$ is irreducible, there exists $0 \neq \lambda \in C$ such that

$$
\sigma \cdot\left(e_{p} \otimes e_{q}\right)=\lambda \cdot I \text {. }
$$

Thus we get that $I \in \operatorname{Hom}_{2}(H, H)$. This implies that $\operatorname{dim} H<\infty$. This finishes the proof.

\section{ACKNOWLEDGMENT}

The author thanks the referee for his suggestions and comments on the revision of this paper.

\section{REFERENCES}

[A] E. Abe, Hopf algebras, Cambridge Univ. Press, London and New York, 1980.

[D] V. Drinfeld, Quantum groups, Proc. Internat. Congr. Math. (Berkeley), Amer. Math. Soc., Providence, RI, 1986, pp. 798-820.

[N] L. Nachbin, On the finite dimensionality of every irreducible unitary representation of a compact group, Proc. Amer. Math. Soc. 12 (1961), 11-12.

[HR] E. Hewitt and K. Ross, Abstract harmonic analysis. II, Springer-Verlag, Berlin, 1970.

[L] R. G. Larson, Characters of Hopf algebras, J. Algebra 17 (1971), 352-368.

[Q] X. Quan, Representations of Hopf $C^{*}$-algebras, preprint.

[W] S. Woronowicz, Compact matrix pseudogroups, Comm. Math. Phys. 111 (1987), 613-665.

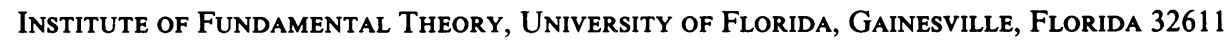

E-mail address: xcq@math.ufl.edu 